\title{
EDIFİCATE
}

I Congreso de Escuelas de Edificación y Arquitectura Técnica de España València, 4 y 5 de noviembre de 2021

Escuela Técnica Superior de Ingeniería de Edificación

Universitat Politècnica de València

Doi: https://doi.org/10.4995/EDIFICATE2021.2021.13591

\section{Proyecto Tetuán como espacio de aprendizaje. Investigación y docencia en Conservación del Patrimonio Edificado.}

\section{Tetouan Project as a learning space. Research and teaching in Conservation of Built Heritage.}

\author{
Lorenzo Jurina ${ }^{a}$, Carlo Manfredi ${ }^{b}$, Carmen Campra García de Viguera y \\ Antonio Jiménez-Delgado \\ aPolitecnico di Milano, Lorenzo.jurina@polimi.it; ${ }^{b}$ Ministero della Cultura, carlo.manfredi@beniculturali.it; \\ 'Universidad de Alicante, Carmen.campra@ua.es; dUniversidad de Alicante, \\ antonio.jimenez@gcloud.ua.es
}

\begin{abstract}
Tetouan Project forms a research and teaching strategy in the field of Conservation of Built Heritage. The physical space of the project is contextualized in the Medina declared in 1997 world heritage by UNESCO that together with the Muslim Cemetery and the Spanish Ensanche draw some of the most beautiful and best preserved landscapes in Morocco.

The project "designs" a series of activities and "develops" them in the space of the Historic Center with the aim of combining the fieldwork of the research group AEDIFICATIO and the teaching of different subjects with undergraduate students in Technical Architecture.
\end{abstract}

Keywords: Tetuán, Tetouan, Medina, preservation, heritage, education, cultural asset, research teaching space, educational project, interdisciplinary work, teaching by research

\section{Resumen}

Proyecto Tetuán configura una estrategia de investigación y docencia en el ámbito de la Conservación del Patrimonio Edificado. El espacio físico del proyecto se contextualiza en la Medina declarada en 1997 patrimonio mundial por la UNESCO que junto al Cemeterio musulmán y el Ensanche español dibujan unos de los paisajes más bellos y mejor conservados de Marruecos. El proyecto "diseña" una serie de actividades y las "desarrolla" en el espacio 
Proyecto Tetuán como espacio de aprendizaje - Tetuan Project as a learning space

del Centro Histórico con el objetivo de combinar el trabajo de campo del grupo de investigación AEDIFICATIO y la docencia de diferentes materias con estudiantes de grado en Arquitectura Técnica.

Palabras clave: Tetuán, Medina, Patrimonio, conservación, educación, bien cultural, espacio docente investigador, proyecto educativo, trabajo interdisciplinar, enseñanza por investigación. 


\section{Introducción}

"Proyecto Tetuán" constituye una estrategia de investigación en herramientas educativas para estudiantes universitarios aplicada a la Conservación del Patrimonio Edificado y su puesta en valor, tanto en exponentes tangibles como en elementos inmateriales del patrimonio cultural.

Desde el ámbito académico, ha sido una oportunidad poder disponer de la Medina de Tetuán como un espacio de investigación para sensibilizar al estudiantado y al profesorado de la UA de la importancia del patrimonio urbano como vehículo de la transmisión cultural y como herramienta educativa para la cooperación internacional.

En 2009 se realiza el "diseño curricular" del proyecto en materia de gestión del patrimonio cultural como línea de investigación experimental dentro del área de conocimiento de Construcciones Arquitectónicas de la UA que serviría para investigar en los sucesivos años hasta la actualidad. La investigación se coordina desde el Grupo de Investigación AEDIFICATIO (Edificación, tecnología, investigación y desarrollo) de la UA. EI "diseño" contempla como principal objetivo definir un modelo de trabajo de investigación donde el/la estudiante participe activamente en el centro histórico de una ciudad que sirve como espacio de aprendizaje e investigación y el/la docente-investigador/a realice el "desarrollo curricular" en el espacio elegido y centre su investigación en dos elementos que conforman un mismo escenario, estudiante y patrimonio cultural utilizando la docencia como objeto de investigación. La ciudad seleccionada es Tetuán, su Medina (declarada en 1997 Patrimonio Mundial por la UNESCO), Cementerio Musulmán y Ensanche Español.

La Medina de Tetuán posee un valor inherente a sus construcciones de gran riqueza arquitectónica. A todo ello se le une de forma inseparable las tradiciones y costumbres de sus habitantes que recorren sus calles a diario.

El análisis de los exponentes constructivos más significativos supone la dinamización de procesos de conservación necesarios en todos los ámbitos de la ciudad. En concreto el caso que se expone sirve como referente para el aprendizaje de un proyecto actual que contemple los objetivos de desarrollo sostenible establecidos por las Naciones Unidas. El proyecto establece las siguientes líneas de trabajo:

- Intervención y conservación en el patrimonio.

- Participación ciudadana.

- Metodología BIM aplicada al patrimonio.

Para una adecuada intervención y conservación del patrimonio se concibe el espacio de la Medina como contenedor de la cultura materializada en sus construcciones en las que reconocemos valores históricos-artísticos.

La participación ciudadana y la inclusión de los datos obtenidos en el proyecto estructura una nueva forma de documento con elementos sociológicos. Las técnicas participativas son nucleares en el proceso. La estratégia metodológica que se plantea es conocida como bottom-up, la cual permite abordar la colectividad desde las diversas y plurales individualidades.

La Medina ha constituido el "espacio docente-investigador" donde desarrollar el trabajo de campo de varias decenas de estudiantes y profesores del área de conocimiento de 
Construcciones Arquitectónicas de la UA en coordinación con expertos y académicos de Tetuán, Granada, Alicante y Milán. La actividad de docencia-investigación supone un modelo cuyos resultados son evaluados en periodos sucesivos.

\section{Objetivos}

Objetivo general: Capacitar de forma integral al estudiante para el trabajo en equipo interdisciplinar, interinstitucional e intergeneracional en materia de "gestión y conservación del patrimonio arquitectónico".

Objetivos particulares:

- Valorar el trabajo en equipo interdisciplinar, interuniversitario e intergeneracional.

- Desarrollar la capacidad de trabajo en un entorno socio-cultural nuevo para el estudiante.

- $\quad$ Poner en valor el análisis de la documentación histórica y sociológica.

- Capacitar en la toma de datos, diagnosis y dictamen en un edificio histórico.

- Desarrollar habilidades en el estudiante para el estudio del patrimonio inmaterial que forma parte del patrimonio arquitectónico y urbano.

- Propiciar el conocimiento del estudiante de las diferentes vías de formación e investigación en el ámbito del patrimonio arquitectónico.

- Desarrollar la capacidad de trabajar en red, finalizado el "workshop", utilizando el capital humano y material del "Proyecto Tetuán" en su futuro profesional y personal.

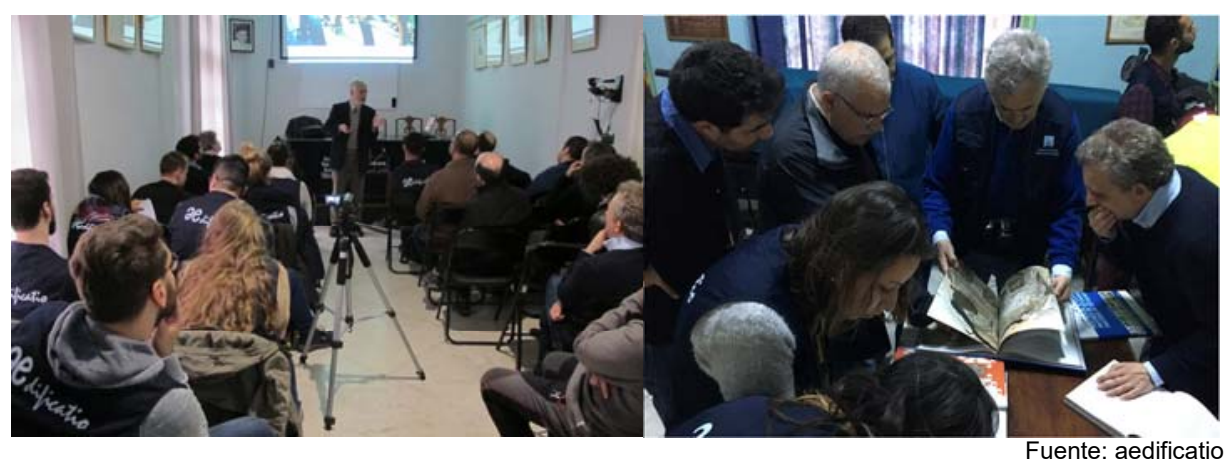

Fig. 1 Actividades de formación e investigación en la sede de la Asociación Tetuán Asmir 


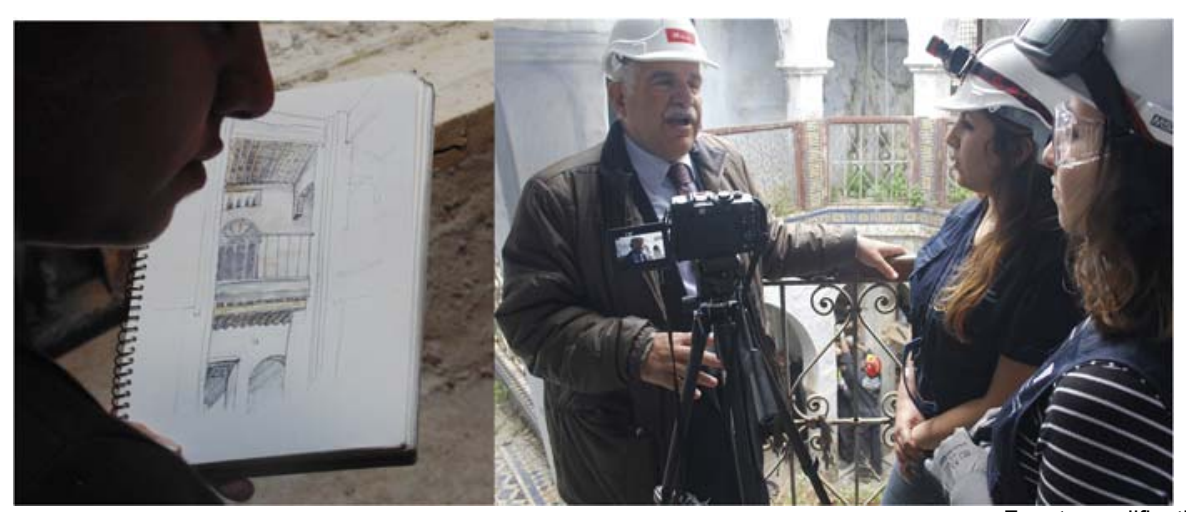

Fig. 2 Trabajo de campo y entrevista a Mohamed Benaboud

Fuente: aedificatio

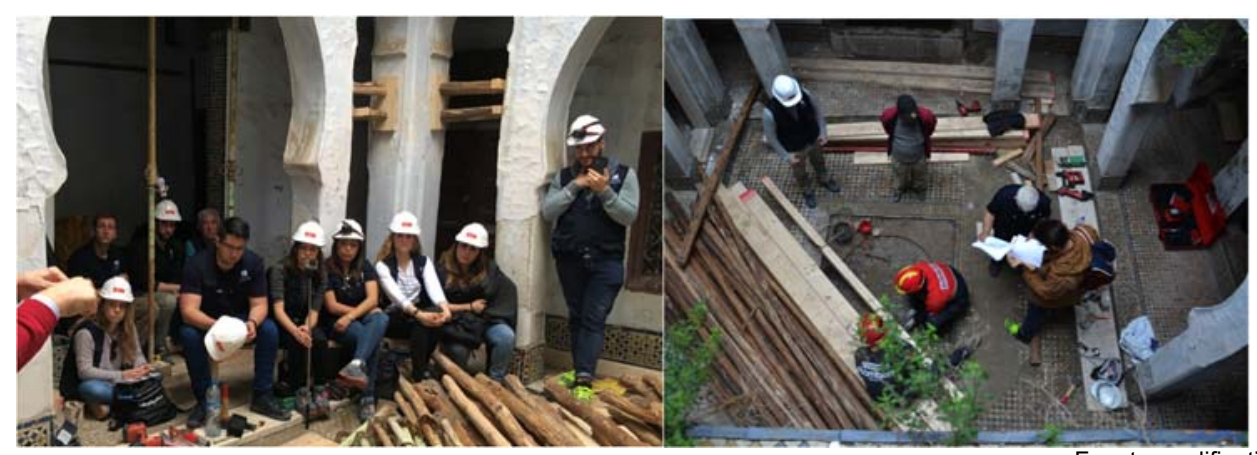

Fig. 3 Clases prácticas in situ e intervención en Casa Ben Marzuk y Mazmorras

\section{Desarrollo de la innovación}

El desarrollo curricular definido en el Proyecto Tetuán establece el método de trabajo y las estrategias para alcanzar los objetivos diseñados con anterioridad. Así, el planteamiento de un "desarrollo" llevado a cabo en los esquemas convencionales del sistema universitario europeo quedan excluidas y se hace necesaria una reflexión profunda de la definición del "espacio" que trasgreda y abra un método adecuado a las necesidades de formación e investigación que la sociedad actual reclama.

En el caso que nos ocupa es necesario establecer un único "método" que responda al desarrollo curricular aglutinando los objetivos diseñados para ambos ejes del Proyecto Tetuán, por un lado la "docencia para el desarrollo del proceso enseñanza-aprendizaje" y por el otro la "investigación sobre el proceso de enseñanza-aprendizaje". Se establece por tanto el siguiente desarrollo:

- Se estudia y se decide un lugar de trabajo que conforma el "espacio" de aprendizaje e investigación con un entorno socio-cultural nuevo para el estudiante.

- Se seleccionan equipos de trabajo interdisciplinar, interuniversitario e intergeneracional. 
- Se busca la documentación histórica y sociológica publicada.

- Se desarrollan actividades de toma de datos, diagnosis y dictamen de edificios históricos y del espacio urbano.

- Se desarrolla una estrategia para el estudio del patrimonio inmaterial que forma parte del patrimonio arquitectónico y urbano.

- Organización de cursos y charlas donde se aborden las vías de formación e investigación en el ámbito del patrimonio arquitectónico.

- Se realiza, para el posterior análisis, material escrito y audiovisual de los diferentes edificios, espacios urbanos y temas abordados del patrimonio tangible e inmaterial.

- Se trabaja en red, finalizado el "workshop", y se realiza un seguimiento de los estudiantes durante los años sucesivos para conocer sus actividades profesionales y personales.

\section{Resultados}

Durante el periodo de trabajo del Proyecto Tetuán se han obtenido numerosos resultados que quedan reflejados en las actividades y documentos generados en los diferentes años de trabajo. En definitiva se han intensificado las relaciones internacionales, los/as estudiantes han desarrollado habilidades y destrezas pluridisciplinares en un ámbito multicultural. Las investigaciones generadas han dado como resultado un riguroso conocimiento del patrimonio construido en la Medina de Tetuán e información inédita de las Mazmorras de la ciudad.

\section{9}

- Trabajo de Campo y toma de datos en la Medina de Tetuán.

- Primera intervención de emergencia en viviendas de La Medina.

- Realización del video: https://www.youtube.com/watch?v=9_-OzYiG6AE.

\section{0-13}

- Trabajo de Campo en la Medina de Tetuán.

- Trabajo de Campo en el Cementerio de Tetuán.

- International Workshop NYC, Milano, Granada y Alicante.

- Sáez Mentxakatorre, I. y Ortega Ortega, L.C. (2012). Guía práctica de Apuntalamientos de emergencia. Alicante: Alarifes.

- Libro: Líndez Vílchez, B. (coord.) (2014). Tetuán, herencia viva. Reflexiones y trabajos resultado de la colaboración de instituciones tetuaníes y la Universidad de Granada.

\section{4-2016}

- $\quad$ Trabajo de campo e investigación en viviendas y espacios de la Medina.

- Trabajo de Campo e investigación en las Mazmorras.

- Intervención de Emergencia en la Casa Ben Marzuk.

- Curso de formación a expertos de Marruecos y España. 


\section{7-18}

- $\quad$ Artículo de investigación: Jiménez Delgado, A., Travaglio, P. y Jurina, L. (2017). Interventi di consolidamento strutturale: il caso delle mazmorras e la casa Ben Marzuk nella Medina di Tétouan. En N. Augenti y L. Jurina (coord.), Atti del convegno IF CRASC'17 (14-16 settembre 2017, Milano) (pp. 543-556). Palermo: Dario Flaccovio Editore.

- Tesis doctoral: Hussey, R.A. (2017). A collaborative rescue archaeological and historical investigation of Tétouan's mazmorras, Morocco (Tesis doctoral). University of Florida. Disponible en http://jurina.it/wp-content/uploads/2013/11/111_Jimenez_A. pdf.

- Producción de vídeos de divulgación científica: ejemplo: http://rua.ua.es/dspace/handle/10045/65309.

\section{9-2021}

- Proyecto Tetuán. Retrospectiva 10 (2009-2019). Exposición retrospectiva fotográfica y audio visual de la actividad investigadora y de intervención en el patrimonio de la Ciudad de Tetuán.

- Libro: Jiménez Delgado, A. (2019). Revoltijo educativo. Pensando la educación. Alicante: Universidad de Alicante.

- $\quad$ Libro: Jiménez Delgado, A., y Lloret, J. (2019). Healt, Wellbeing and Sustainability in the Mediterranean City: Interdisciplinary Perspectives. New York-London: Routledge.

\section{Conclusiones}

El Proyecto Tetuán, al igual que otros proyectos realizados con anterioridad, han servido de base para establecer un método de trabajo en el denominado "Proyecto Aedificatio" que se centra en la construcción y conservación del patrimonio arquitectónico. Se desarrolla de forma interdisciplinar, tomando al ser humano y el bienestar social como centro y con un objetivo didáctico y cultural. Es un proyecto sostenible que une la arquitectura contemporánea y la tecnología con la tradición y el saber vernáculo, teniendo en cuenta la historia y el genius loci.

Los miembros del grupo de investigación "Aedificatio" están unidos por el interés hacia un trabajo profesional, de investigación y reflexión cultural basado en los siguientes valores compartidos. El grupo hace participe en sus actividades a estudiantes para formentar la docencia de calidad junto a la investigación.

Una vez desarrollado el "Proyecto Aedificatio" llevado a cabo en el contexto de la ciudad de Tetúan enumeramos las siguientes conclusiones de la actividad de aprendizajeinvestigación: 
Se basa en la interdisciplina, creyendo en la necesidad de superar los límites de un saber monodisciplinar, anclado en la singularidad de cada disciplina, en favor de la cooperación entre especialistas procedentes de distintos ramos del saber: arquitectura, ingeniería, sociología, historia, historia del arte, tecnología...

Propicia el encuentro y debate constructivo y formativo por parte de los diferentes miembros del grupo de investigación, el compromiso activo e intercambio de ideas.

Tiene como centro al ser humano, ententidiendolo como singularidad y colectividad. Hoy en día, frente a un mundo nuevo, cada vez más multicultural, multiétnico y multireligioso, se hace necesario un "Nuevo Humanismo" como redescubrimiento del hombre y de su relación con la realidad en la cual vive, como reafirmación del valor de la persona.

Otro eje es el bienestar social. El proyecto se basa en dar respuestas a las necesidades de la colectividad. Para ello es imprescindible el contacto directo con la población, propiciando la participación ciudadana y analizando la información recibida para un adecuado diagnóstico.

Tiene como objetivo generar y fomentar la cultura, produciendo un contenido que favorezca un pensamiento critico y reflexivo. Es un proyecto global e intercultural en cuanto a visión, expertos y metodología.

Otro objetivo es la educación. El "Proyecto Aedificatio" tiene una dimensión didáctica, propiciando la explicación y la difusión de contenidos dirigidos a la educación primaria, secundaria y universitaria. La misma palabra "Aedificatio" contiene en si este concepto fundamental: del lat. aedificare, con el significado de construir y fundar, si no también de educar, instruir, iluminar. El "Proyecto Aedificatio" tiene en cuenta la misión de la UNESCO en cuanto a Educación, Ciencia y Cultura. La educación y difusión, junto con el pensamiento crítico y reflexivo que se pretende alcanzar, llevan al empleo de un método de enseñanza por investigación o "problematizado"; basado en la sucesión de preguntas presentadas como problemas que el alumno debe ir cuestionándose y resolviendo por sí mismo mediante la autoreflexión.

Pretende fomentar la curiosidad y creatividad del observador así como propiciar la belleza y armonía del mismo diseño del proyecto arquitectónico y de su contenido, activando cada uno de los puntos desarrollados en el presente manifiesto.

Contempla los 17 objetivos de desarrollo sostenible de la ONU.

Une la contemporaneidad y la tecnología más avanzada con la tradición y saber vernáculo, enfatizando los elementos locales así como los globales y visiones intergeneracionales.

Contempla como objetivo último la preservación de los elementos indentitarios del lugar en el cual se interviene y la puesta en valor el genius loci. 
Lorenzo Jurina, Carlo Manfredi, Carmen Campra García de Viguera y Antonio Jiménez-Delgado

\section{Referencias}

ABDERRAHMAN (2015), El Fathi: Marruecos en Cervantes. Antología de textos. Patio de Monipodio, Tetuán.

BENABOUD, M. (coord.) (2004) Tétouan, capitale méditerranéenne, Asociación Tétouan-Asmir, Tétouan.

ERZINI, N. (2015), La influencia renacentista y mudejár en la arquitectura doméstica de Tetuán en los siglos XVI y XVII. Los Moriscos-Andalusies en Marruecos. Estado de la cuestión. Actas del Coloquio Internacional, Asociación Marroquí de Estudios Andalusies, pp. 41-64.

GÓMEZ MORENO, M. (1922) Descubrimientos y antigüedades en Tetuán. Boletín Oficial de la Zona de Protectorado Español en Marruecos, suplemento 10.

GOZALBES BUSTO, G. (1984) Las mazmorras de Tetuán. Estudios de Historia y Arqueología Medievales, 3-4, pp. 247-264.

GOZALBES BUSTO, G (1992) Los moriscos en Marruecos. Guillermo Gozalbes Busto, Granada.

GOZALBES, E. (2012) Tetuán: arqueología, historia y patrimonio. Asociación Tetuán-Asmir, Tetuán.

MIEGE, J.L., BENABOUD, M., ERZINI, N. (1996) Tétouan: ville andalouse marocaine, CNRS-Paris, Kalila Wa Dimna-Rabat.

MONTALBÁN Y MAZAS, C.S. (1939) Las mazmorras de Tetuán, su limpieza y exploración, Editorial Mundo Latino, Madrid.

Primo volume delle navigationi et viaggi nel qual si contiene la Descrittione dell'Africa (1550) Heredi di Lucantonio Giunti, Venezia, 1550.

SORINO, G., JIMÉNEZ-DELGADO, A., MANFREDI,C. (2019) BIM Methodology Applied To Architectural Heritage Preservation: Case Study Of The Medina And Dungeons Of Tetuán, Morocco, in "Building Information Modelling (BIM) in Design, Construction and Operations III", Edited By: De Wilde, P., Mahdjoubi, L., Garrigós Garrigos, A. Wit Press, Ashurst, pp. 157-168

TORRES LÓPEZ, R. (2011) La Medina di Tetuán. Guía de arquitectura, Conserjeria de Obras Públicas y Vivienda-Sevilla,Consejo Municipal de Tetuán-Tetuán, 3a edizione. 